\title{
Internacionalización y supervivencia exportadora. Un estado de la cuestión
}

\author{
Internationalization and export survival. The state of the question
}

\author{
Rogelio JORGE-MARTÍN \\ Programa de Doctorado en Historia \\ e Historia del Arte y Territorio \\ Universidad Nacional de Educación \\ a Distancia (UNED) \\ rogjorge@ponferrada.uned.es \\ www.rogeliojorge.es
}

\begin{abstract}
Resumen: El proceso de internacionalización ha sido objeto de numerosas investigaciones. El presente artículo ofrece una breve visión de conjunto de la teoría actual acerca de la internacionalización y la decisión empresarial de llevarla a cabo. Desde una perspectiva histórica se presenta una breve definición del concepto. Además, se analiza la decisión de internacionalización, sus razones, condicionantes, la actitud necesaria y los efectos que tiene.
\end{abstract}

Abstract: Internationalization has been the subject of several investigations. This article presents an overview of the theory of internationalization and the pertaining business decision. A historical perspective is followed by a brief definition of the term. The decisions, reasons, conditions and necessary attitude, as well as the effects, are analyzed thereafter.

Palabras clave: internacionalización, exportación, supervivencia exportadora, regularidad exportadora, comercio internacional, comercio exterior, barreras a la exportación, plan de internacionalización, estrategia de internacionalización, diversificación de mercados.

Keywords: internationalization, export, export survival, export regularity, international trade, foreign trade, export barriers, internationalization plan, internationalization strategy, market diversification.

\section{Sumario:}

\section{Introducción.}


II. La internacionalización: perspectiva e historia.

2.1. Perspectiva histórica del comercio exterior.

2.2. El concepto de internacionalización y de exportación

III. La decisión de la internacionalización.

3.1. Razones para la internacionalización.

3.2. Condicionantes de la internacionalización de las pymes.

3.3. Dos actitudes frente a la internacionalización: proactiva y reactiva.

3.4. Efectos de la internacionalización.

IV. Conclusiones.

V. Bibliografía.

Recibido: septiembre 2021.

Aceptado: noviembre 2021. 


\section{INTRODUCCIÓN}

La internacionalización es el objetivo final de las empresas que buscan ampliar su mercado, independientemente de su tamaño. Es un proceso costoso y a largo plazo que requiere una inversión de recursos y tiempo. Muchas empresas fracasan en este proyecto, sobre todo por la falta de planificación y por tener una actitud equivocada. Las empresas que tienen éxito y sobreviven como exportadoras se benefician de toda una serie de ventajas brindadas por la ampliación y diversificación de mercados y por la exigencia de profesionalización y adaptación que supone internacionalizarse.

\section{LA INTERNACIONALIZACIÓN: PERSPECTIVA E HISTORIA}

Para poder tener una visión general de lo que es la internacionalización en el ámbito de las empresas es necesario tener una perspectiva histórica del comercio exterior. También es preciso definir claramente el concepto de internacionalización pues, como se verá más adelante, no es algo sobre lo que exista unanimidad.

\subsection{Perspectiva histórica del comercio exterior}

Al hablar de la internacionalización de las empresas es inevitable hacer referencia a dos términos: empresario y globalización. En el siglo XVI se empezó a utilizar el término francés entrepreneur (emprendedor) para referirse a individuos que lideraban operaciones militares. El término se relacionó con aquellas personas de carácter aventurero y con cierta propensión a correr riesgos en la búsqueda de beneficios, con independencia económica $\mathrm{y}$, ante todo, capacidad gestora y de organización de actividades y personas ${ }^{1}$. En cuanto a la globalización, Ramón Tamames señala que el fenómeno cuenta con "[...] muy remotos antecedentes, cuando menos desde el Tratado de Tordesillas de 1494 entre España y Portugal para el reparto del globo"2. Tras este tratado,

\footnotetext{
${ }^{1}$ GARRIDO BUJ, S., Manual de gestión de empresas. Madrid, Editorial Universitas UNED, 2011.

2 TAMAMES, R., "Sin alternativa a la globalización", en Revista de economía mundial, 18 (2008) 138 .
} 
destaca el hito de la circunnavegación de la tierra por la expedición MagallanesElcano entre 1519 y $1522^{3}$.

El monopolio comercial, la prohibición del tráfico libre de metales preciosos y otros productos, y la reglamentación directa del comercio fueron los pilares básicos que se sustentaron el mercantilismo de los siglos XVI al XVIII. En este marco, el desarrollo de las manufacturas se basaba en los monopolios y el comercio quedó asociado a las colonias ${ }^{4}$. Cada potencia europea tenía su propia zona de influencia, delimitada por sus posesiones ultramarinas.

El comercio mundial se formó gracias al surgimiento del capitalismo comercial entre los siglos XVI y XVII. Éste produjo un aumento de la producción de bienes y del tráfico marítimo entre América y los principales puertos tanto del Mediterráneo como del norte de Europa. El principio de balanza comercial favorable fue el pilar básico del mercantilismo entre los siglos XVI y XVIII. El mercantilismo se basaba en rechazar la exportación de materias primas y preferir la de productos manufacturados o elaborados con un valor mayor. Las importaciones debían reducirse a las imprescindibles, y siempre bajo la premisa de menor valor. Respecto a los productos de lujo, por lo general se prohibió o restringió su importación ${ }^{5}$.

Entre los más destacados autores mercantilistas se encuentra el economista inglés Thomas Mun. En su obra La riqueza de Inglaterra por el comercio exterior, respecto al valor del comercio exterior, afirmaba lo siguiente:

"[...] Considerad, pues, la verdadera forma y valor del comercio exterior, el cual es: la gran renta del rey, la honra del reino, la noble profesión del comerciante, la escuela de nuestros oficios, la satisfacción de nuestras necesidades, el empleo de nuestros pobres, el mejoramiento de nuestras tierras, la manutención de nuestros marineros [...]. Por todas estas grandes y poderosas razones muchos estados bien gobernados fomentan grandemente esta profesión y cuidadosamente estimulan esta actividad, no solamente con una política que la aumente, sino también con poder para protegerla de daños externos $[\ldots]^{\prime \prime 6}$.

\footnotetext{
${ }^{3}$ PIGAFETTA, A., La primera vuelta al mundo: Relación de la expedición de Magallanes y Elcano (1519-1522). Madrid, Alianza Editorial, 2019.

4 CALVO, A., Economía internacional y organismos económicos internacionales, 2. Madrid, Editorial Universitaria Ramón Areces UNED, 2016.

${ }^{5}$ CALVO, A., Economía internacional..., o.c.

${ }^{6}$ MUN, T., "La riqueza de Inglaterra por el comercio exterior", en Fondo de cultura económica $(1664 / 1978)$, p. 3.
} 
Además, ofrecía las guías a seguir en dicho comercio:

"[...] para aumentar nuestra riqueza y tesoro son por el comercio exterior, por lo que debemos siempre observar esta regla: vender más anualmente a los extranjeros en valor de lo que consumimos de ellos [...] porque la parte de nuestro patrimonio (exportaciones) que no nos sea devuelta en mercaderías (a través de las importaciones) debe necesariamente regresar en dinero $[\ldots]^{3}$.

También remarcaba la importancia de que Inglaterra utilizara sus propios medios para todo: mercancía, transporte y seguros. Así se incrementaría el valor de la exportación.

En 1776 la obra de Adam Smith, La riqueza de las naciones, marcó un punto de inflexión. Provocó las primeras grietas del sistema mercantilista y su autor emergió como representante fundamental de la teoría económica clásica. A pesar de que había antecedentes de sus ideas, como los trabajos de David Hume, fue Adam Smith quien lideró la crítica al sistema de monopolio de exportación y el proteccionismo y esbozó las ventajas de un comercio universal sin barreras. A finales del siglo XVIII el mercantilismo fue dejando paso a una postura más librecambista. En esta época la estructura económica se basaba principalmente en pequeñas empresas de tipo familiar en las que las figuras del empresario y del propietario eran la misma persona. Fue un momento en que se abrieron las relaciones entre países en el ámbito del comercio exterior. Con esta apertura se fueron desarrollando las teorías del comercio internacional ${ }^{8}$.

Con la aparición de la máquina de vapor y el carbón como fuente de energía básica a finales del XVIII y principios del XIX, nació la Revolución Industrial y con ella la nueva empresa capitalista. En este momento, la figura de empresario-propietario, que hasta entonces unificaba capital y recursos humanos, empezó a ser sustituido por organizaciones con gestores, independientemente de quién fuera el propietario9.

En Inglaterra los beneficios derivados del librecambismo, el intervencionismo del Estado en la industria y el comercio junto a la industrialización, hicieron que este país jugara un papel predominante en el entramado teórico sobre el comercio internacional y los beneficios derivados del mismo. El resto de los países europeos siguieron su estela con más o menos éxito. Para Adam Smith, a finales

\footnotetext{
7 ÍDEM, p. 2.

${ }^{8}$ TAMAMES, R., Sin alternativa..., o.c.; GARRIDO BUJ, S., Manual de gestión ..., o.c.; CALVO, A., Economía internacional..., o.c.

${ }^{9}$ GARRIDO BUJ, S., Manual de gestión ..., o.c.
} 
del siglo XVIII, el comercio se limitaba a la aplicación del principio de la división del trabajo ${ }^{10}$. En cierta manera, para Smith, tanto el comercio internacional como el comercio doméstico se desarrollan de la misma manera y bajo las mismas reglas. Cada país podrá producir y comerciar el bien en cuya producción tuviera una ventaja en términos de coste, es decir, producía lo mismo con menos recursos (teoría de la ventaja absoluta). La teoría de Adam Smith dio paso a la teoría de la ventaja comparativa de David Ricardo que posteriormente ampliarían autores como John Stuart Mill. Este último señala que los beneficios indirectos del comercio internacional, tanto económicos como morales, son incluso mayores que los directos ${ }^{11}$.

En 1817 David Ricardo ofreció una explicación al comercio y demostró que el comercio internacional no tiene que estar necesariamente basado en la teoría de la ventaja absoluta, como mantenía Adam Smith. Ricardo desarrolló la teoría de la ventaja comparativa:

“[...] Inglaterra exporta tejidos a cambio de vino, porque, obrando así, resulta más productiva su actividad industrial; obtiene así más tejidos y más vino de los que obtendría produciendo ambas cosas ella misma, y Portugal importa tejidos y exporta vino porque su actividad le rinde más provecho produciendo vino para los dos países"12.

Otro concepto importante introducido por David Ricardo es el de coste de oportunidad. Señala que, si un país quiere producir dos bienes, tendrá que renunciar a producir determinadas unidades de uno de ellos para producir el otro, y viceversa ${ }^{13}$.

La teoría de la ventaja comparativa es importante para comprender cómo funciona el comercio internacional. No obstante, se puede considerar una teoría sesgada por el lado de la oferta. Esta teoría fue ampliamente analizada por autores posteriores que introdujeron en el análisis del intercambio las condiciones de la demanda, representaron gráficamente la teoría ricardiana, expusieron lo grandes que pueden ser las ganancias del comercio internacional, incluyeron la idea de coste de oportunidad y señalaron la importancia de considerar la demanda de forma global ${ }^{14}$.

\footnotetext{
${ }^{10}$ SMITH, A., La riqueza de las naciones. Madrid, Alianza Editorial, 1776/1997.

${ }^{11}$ MILL, J. S., Principles of Political Economy. New York, D. Appleton and Company, 1848/1885.

12 RICARDO, D., Principios de Economía Politica y Tributación. Madrid, Ediciones Pirámide, 1817/2003, p. 120.

${ }^{13}$ KRUGMAN, P. R., y OBSTFELD, M., Economía internacional. 7. Madrid, Pearson Educación, 2006.

${ }^{14}$ MARSHALL, A., Principles of Economics. 8a . London, MacMillan, 1890/1920; EDGEWORTH, F. Y., Mathematical Psychics an Essay on the Application of Mathematics to the Moral Sciences,
} 
En los años 30 del siglo XX, surgió la teoría de Eli Heckscher y Bertil Ohlin ${ }^{15}$. Según estos autores el comercio tiene lugar porque los países tienen diferente dotación de factores. Existen países con abundancia de mano de obra, que exportarán bienes intensivos en trabajo, y países con abundancia de capital, que exportarán bienes intensivos en capital. Esta teoría está basada en el supuesto de la competencia perfecta, sin obstáculos al comercio, sin costes de transporte y bajo patrones de consumo similares entre los países. Fue discutida por autores como Ronald Jones y J. Peter Neary y economistas como Harry G. Johnson, Kelvin Lancaster, Wolfgang Stolper y Paul Anthony Samuelson, que completaron el análisis ${ }^{16}$.

La nueva teoría del comercio internacional tiene sus comienzos con autores como Paul Krugman, quien a través de sus trabajos en los años 70 y 80 consideró enfoques nuevos que permiten entender mejor el comercio entre países, como la optimización de costes en las economías de escala ${ }^{17}$. De esta forma cada país se puede especializar en la producción de ciertos bienes y comprar el resto a otros países, aprovechando las economías de escala. Esto ayuda a diversificar la oferta e incluso países que son suministradores pueden también ser consumidores del mismo producto que proviene de un país distinto. Con ello se da origen a un nuevo enfoque del comercio internacional.

En los años 90 destaca Michael Porter y su concepto de ventaja competitiva. Para Porter existen dos ventajas competitivas fundamentadas: los bajos costes y la diferenciación. Estas dos ventajas derivan en dos estrategias entre las cuales la empresa tendrá que decidir. Para hacerlo debe tener en cuenta el análisis de su cadena de valor ${ }^{18}$. Existen sectores, como el vitivinícola, donde, partiendo de un producto, las empresas pueden adoptar una estrategia $u$ otra, dependiendo del lugar que ocupe cada bodega y del valor añadido que deseen o puedan incorporar al producto ${ }^{19}$.

Forgotten Books, 1881/2012. HABERLER, G., The theory of international trade. London, Willian Hodge, 1936; LEONTIEF, W. W., "The use of indifference curves in the analysis of foreign trade", in The quarterly Journal of Economics, 47, 3 (1933) 493-503.

${ }^{15}$ HECKSCHER, E. F., y OHLIN, B.G., Heckscher-Ohlin trade theory, The MIT Press, 1991.

${ }^{16}$ JONES, R. W., y NEARY, J.P., "The positive theory of international trade". JONES, R. W., y KENEN, P.K., Handbook of international economics, Vol. 1, Amsterdam, North-Holland, 1984; JOHNSON, H.G., International trade and economic growth: studies in pure theory. Harvard University Press, 1958; LANCASTER, K., "Intra-industry trade under perfect monopolistic competition", in Journal of International Economics, 1980, 151-175; STOLPER, W. F., y SAMUELSON, P.A., "Protection and real wages", in The review of economic Studies, 9.1 (1941) 58-73; SAMUELSON, P. A., "The gains from international trade one again", in Economic Journal, 72.288 (1962) 820-829.

${ }^{17}$ KRUGMAN, P. R., \& OBSTFELD, M., Economía internacional..., o.c.

18 PORTER, M. E., Ventaja competitiva. Madrid, Alay, 2002.

${ }^{19}$ JORGE-MARTÍN, R., "El arraigo territorial en la internacionalización del vino español", en Anuario Jurídico y Económico Escurialense, 53 (2020) 353-378. 


\subsection{El concepto de internacionalización y de exportación}

A priori parece que no hay un consenso claro acerca de la definición exacta de internacionalización y es fácil confundirla con la exportación. Conceptualizar o elaborar una definición clara no es fácil. En general, se puede considerar que para una empresa la internacionalización es un proceso con varios grados o estadios, que se realiza en cualquier operación en su cadena de valor de forma regular fuera de su país de origen y alejado de su entorno geográfico natural, entendiendo como tal la zona donde la empresa opera como si se tratara de un mercado doméstico. Exige el compromiso de dedicar recursos, tanto económicos como humanos, a medio y largo plazo en el país de destino. En definitiva, es un paso más que la mera importación o exportación ${ }^{20}$.

Este proceso debería llevar asociado la elaboración de un Plan de Internacionalización. Si no hay recursos comprometidos y lo que se hace es abastecerse o vender en el exterior, sería simplemente una actividad internacional de importación o exportación, que frecuentemente es el punto de partida para una futura internacionalización ${ }^{21}$.

La mayoría de los procesos de internacionalización han seguido una serie de etapas secuenciales hasta adquirir el grado deseado por la empresa. Los modelos de internacionalización más conocidos son el modelo nórdico de la Escuela Uppsala y el modelo estadounidense Bilkey \& Tesar (Véanse figuras 1 y 2).

Figura 1: Modelo de internacionalización de la Escuela Uppsala

\begin{tabular}{|c|l|}
\hline FASE & MODELO ESCUELA UPPSALA (modelo nórdico) \\
\hline 1 & Exportador esporádica e intermitente \\
\hline 2 & Exportador vía agentes \\
\hline 3 & Exportador vía filiales de venta \\
\hline 4 & Establecimiento de subsidiarias de producción en el exterior \\
\hline
\end{tabular}

Fuente: Johanson y Wiedersheim-Paul (1975). Elaboración propia

${ }^{20}$ VAHLNE, J. F., y JOHANSON, J., "The Uppsala model: Networks and microfundations", in Journal of International Business Studies, 51,1 (2020) 4-10; CARNICER, D., Price in international marketing, Madrid, ESIC Editorial, 2016; ICEX-CECO, Manual de internacionalización, 2. Madrid, AEBOE, 2017. JOHANSON, J., y VAHLNE, J.F., "The internationalisation process of the firm. A model of knowledge development and increasing foreign market commitments", in Journal of International Business Studies, 8,1 (1977) 23-32; CAVUSGIL, S. T., Organizational determinants of the firm's export behavior: an empirical analysis. Ph. D. dissertation. The University of Wisconsin, 1976.

${ }^{21}$ VARIOS, "Explaining intermittent exporting: exit and conditional re-entry in export markets”, in Journal of International Business Studies, 47, 9 (2016) 1058-1076. 
Figura 2: Modelo de internacionalización de Bilkey y Tesar

\begin{tabular}{|c|l|}
\hline FASE & MODELO BILKEY \& TESAR (modelo estadounidense) \\
\hline 1 & Exportador potencial, pero no exporta \\
\hline 2 & Exportador ocasional, pedidos inesperados \\
\hline 3 & Exportador esporádico que activamente explora la posibilidad de exportar \\
\hline 4 & Exportador experimental a mercados cercanos psicológicamente. \\
\hline 5 & Exportador regular en un mercado \\
\hline 6 & Exportador regular y diversificación a mercados lejanos \\
\hline
\end{tabular}

Fuente: Johanson y Wiedersheim-Paul (1975) y Bilkey y Tesar (1977).

Elaboración propia

Las empresas no tienen necesariamente que llegar a los estadios más altos del proceso de internacionalización, pero sí al mínimo de ser exportadoras regulares para tener una actividad sostenible en el tiempo y sobrevivir como exportadoras.

\section{LA DECISIÓN DE LA INTERNACIONALIZACIÓN}

Tomar la decisión de internacionalizarse no es algo que se pueda hacer a la ligera. Las empresas deben tener en cuenta toda una serie de aspectos que influyen en esta decisión. Lo primero que se debe comprender es que existen diferentes razones para llevar a cabo este proyecto. La internacionalización está condicionada por múltiples factores dentro y fuera del control de la empresa. Ante esta situación se pueden adoptar dos actitudes: proactiva o reactiva.

\subsection{Razones para la internacionalización}

Los pilares del espíritu emprendedor son la figura del empresario e innovador con sensibilidad hacia el desarrollo de nuevos productos y la apertura de nuevos mercados, el acceso a nuevas fuentes de aprovisionamiento o a nuevos procedimientos de dirección y organización. Junto a estos pilares hay que añadir los conceptos de riesgo e incertidumbre empresarial ${ }^{22}$.

Para la empresa es de suma importancia conocer los motivos para iniciar y desarrollar la actividad internacional. Las razones para la internacionalización radican en diversos motivos que pueden variar a lo largo del tiempo en un entorno

${ }^{22}$ SCHUMPETER, J. A., Theorie der wirtschaftlichen Entwicklung, Leipzig, Verlag von Duncker und Humblot, 1911. 
de globalización de mercados ${ }^{23}$. En función de esas razones se internacionalizará o se limitará a exportar temporalmente. De ellas dependerán aspectos como el nivel de internacionalización deseado o los países en los que se actuará. El conocimiento por parte de cada empresa de estas causas para la actividad internacional no está solamente destinado a las que van a iniciarse, sino también a exportadoras activas que pretenden reorientarse o expandirse a otros mercados.

Entre las razones que llevan a la empresa a plantearse la internacionalización el ICEX (Instituto Español de Comercio Exterior) señala que las principales son: reducir los costes de producción, acceder a nuevos recursos y acceder a nuevos mercados ${ }^{24}$. Esto permite aclarar cuál es la relación entre exportación e internacionalización. La exportación puede ser una de las actividades dentro de la internacionalización. De hecho, es la más común en el caso de España. Sin embargo, ni la internacionalización exige una actividad exportadora, porque una empresa puede producir en otro país y vender allí lo producido; ni la exportación requiere la internacionalización de la empresa, porque una empresa puede exportar puntualmente (este suele ser el inicio de la internacionalización). La exportación es una de las formas más fáciles de entrada en los mercados internacionales porque implica un riesgo mínimo, una inversión económica y humana reducidas, y una gran flexibilidad.

En el trasfondo de la decisión de internacionalizarse o salir a los mercados exteriores hay una serie de motivaciones que se pueden dividir en proactivas y reactivas. Las primeras están relacionadas con las fortalezas internas de las empresas, como puede ser poseer una ventaja competitiva o bien por oportunidades en mercados exteriores. Las segundas se deben a amenazas que acechen a la empresa o bien a debilidades internas. Para detectar estos factores es imprescindible hacer un buen análisis DAFO (debilidades, amenazas, fortalezas y oportunidades) antes de internacionalizarse de forma improvisada ${ }^{25}$.

El deseo o necesidad de acceder a nuevos mercados es una de las razones más habituales de las empresas para internacionalizarse. Ya sea por compensar una bajada en la demanda doméstica o porque un producto concreto entre en su fase de madurez o esté llegando a la de declive, la búsqueda de otros mercados es una razón de peso para iniciar las primeras fases de la internacionalización ${ }^{26}$.

${ }^{23}$ VARIOS, "Marketing internacional". Manual de internacionalización, 2. Madrid, ICEX España, Exportación e Inversiones y el Centro de Estudios Económicos y Comerciales, 2017, pp. 218-372.

${ }^{24}$ ICEX-CECO, Manual de internacionalización..., o.c.

${ }^{25}$ HOLLENSEN, S., y ARTEAGA, J., Estrategia de marketing internacional, 4. Madrid, Pearson Educación, 2010.

${ }^{26}$ ICEX-CECO, Manual de internacionalización..., o.c.; REQUEIJO, J., "La competitividad de la economía española (2008-2018)”, en Economistas, 166 (2020) 31-36. 
La historia reciente de la actividad internacional de las pymes españolas ha tenido un denominador común. Muchas pymes han salido al exterior en busca de oportunidades cuando la demanda interna flaqueaba y es entonces cuando recurrían a mercados exteriores para contrarrestar esta situación. Lo más desalentador es que parte de esas empresas que iniciaban su andadura internacional la abandonaban al recuperarse la demanda interna ${ }^{27}$.

Partiendo de la premisa de que se cuente con un producto exportable, tanto física como económicamente, la empresa exportadora, sobre todo en sus inicios, debe realizar una selección sistemática de los países potenciales a los que podría exportar. También tiene que seleccionar qué productos desea exportar y elegir la mejor forma para hacerlo determinando un precio apropiado. La mejor manera de afrontar este proceso es contar con un plan de internacionalización y barajar la mejor opción de penetración en el mercado objetivo, teniendo en cuenta los recursos disponibles, el nivel de implicación deseado y la forma de acceso. Mientras que los problemas de gestión se encuentran a lo largo de todo el proceso, los relacionados con el marketing y el marketing internacional aparecen fundamentalmente en las fases iniciales ${ }^{28}$.

Las empresas están en un entorno en el que la demanda está cada vez más globalizada y que en general acorta el ciclo de vida de los productos. El desarrollo de cualquier producto conlleva una fuerte inversión en tiempo y en recursos, sobre todo en sus primeras fases. Por otro lado, la venta de un producto en nuevos mercados puede alargar su ciclo de vida al llegar a segmentos, canales o nuevos clientes, incluso en el caso de productos en la fase final de declive. Cualquier posibilidad de alargar el ciclo de vida de un producto es una buena oportunidad para las empresas y una motivación más para salir a los mercados internacionales ${ }^{29}$.

No hay una medida universal para determinar el grado de internacionalización. En empresas multinacionales se debe basar en tres factores: generación de

${ }^{27}$ BONET, A., "La nueva Cámara de Comercio de España y la internacionalización empresarial", en Economistas, 148 (2016) 157-165.

${ }^{28}$ SECRETARÍA DE ESTADO DE COMERCIO, Plan de acción para la internacionalización de la economía española 2021-2022, Madrid, CPAGE, 2020; CARNICER, D., Plan de internacionalización de empresas, Valencia, Centros Europeos de Empresas Innovadoras de la Comunidad Valenciana (CEEI CV), 2011; CARNICER, D., "Claves para la Internacionalización de la Empresa Familiar", en Cuadernos de Trabajo de la Cátedra de Empresa Familiar de la Universitat de València, Valencia 2020; AABY, N., y SLATER, S.F., "Management influences on export performance: a review of the empirical literature 1978-1988", in International marketing review, 6, 4 (1989) 7-26.

${ }^{29}$ ICEX-CECO, Manual de internacionalización..., o.c.; CARNICER, D., Price in international..., o.c. 
ventas, activos en el extranjero y la dispersión geográfica de las actividades internacionales. Por ello se utilizan distintos índices. Hay estudios que se centran en las características y presencia de empresas en actividades internacionales a través de filiales y en zona europea. Los hay que analizan el índice de internacionalización en base al número de países en los que opera una empresa. $\mathrm{Y}$ otros han tratado las cuestiones que hay detrás de la evaluación del grado de internacionalización de las empresas ${ }^{30}$.

Sin embargo, el aspecto más importante para determinar el grado de internacionalización es el crecimiento de la exportación y su valor sobre el total de las ventas de la empresa. Por otra parte, también es relevante el nivel de diversificación de mercados. En definitiva, puede afirmarse que el grado de internacionalización viene indicado por cuatro factores principales: número de países en los que la empresa vende, diversificación de zonas geográficas, porcentaje de ventas exteriores sobre el total y los recursos comprometidos en los mercados internacionales.

Figura 3: Elementos para determinar el grado de internacionalización de la empresa

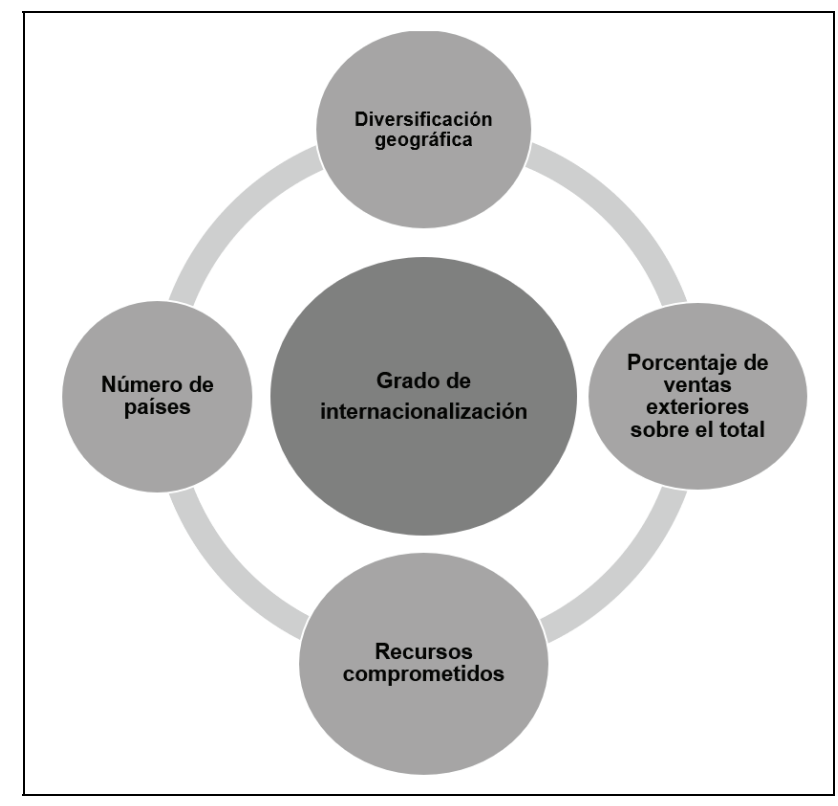

Elaboración propia.

${ }^{30}$ IETTO-GILLIES, G., "Conceptual issues behind the assessment of the degree of internationalization", in Transnational Corporations, 18, 3 (2009). 


\subsection{Condicionantes de la internacionalización de las pymes}

Los factores que influyen en la internacionalización de las empresas son muchos y muy variados. La Comisión Europea establece una clasificación identificando dos tipos de factores. Los primeros externos y los segundos internos ${ }^{31}$. Los factores externos incluyen los relacionados con el país de destino y los relacionados con el país del exportador. Los factores internos son los relacionados con los recursos de la empresa y los relacionados con el marketing. Se pude distinguir si un factor es externo o interno porque la empresa no tiene influencia sobre los factores externos y sí sobre los internos.

Los condicionantes externos son los que no pueden ser controlados o influenciados directamente por las empresas, sino que son producto de factores externos y el entorno ${ }^{32}$. Los hay de dos categorías: los relacionados con el país de destino y los relacionados con el país del exportador. Los primeros incluyen los derivados del mercado, político-legales, económicos y socioculturales. Entre los segundos se encuentran los de tipo gubernamental, sectorial y geográfico.

Por lo general, los factores relacionados con el entorno del país de destino tienen una repercusión rápida y una gran dificultad de ser predichas con anterioridad, afectando a exportadores noveles, intermitentes y regulares ${ }^{33}$. Dentro de esta categoría hay varios tipos de condicionantes de la exportación: de mercado, político-legales, económicas y socioculturales. Las que mayor impacto tienen son las relacionadas con el mercado, legal y político.

Los condicionantes externos no se encuentran solo en el país de destino de las exportaciones. También los hay relacionados con el entorno del país de origen del exportador y la mercancía ${ }^{34}$. Estas pueden dividirse en tres tipos. En primer lugar, las derivadas de la acción del Estado del país. En segundo lugar, los relacionadas con el sector económico al que pertenece el exportador. Y, por último, los derivados de la ubicación geográfica de las empresas exportadoras y la mercancía.

${ }^{31}$ COMISIÓN EUROPEA, "Drivers of SME internationalization”, 2014.

32 ARTEAGA, J., La actividad exportadora y las barreras a la exportación. Una aplicación empírica a las pequeñas y medianas empresas españolas. (Tesis doctoral). Universidad de Las Palmas de Gran Canaria, 2003.

${ }^{33}$ CRICK, D., "The decision to discontinue exporting: SMEs in two U.K. trade sectors.", in Journal of Small Business Management, 40,1 (2002) 66-77; LEONIDOU, L. C., "An analysis of the barriers hindering small business export development", in Journal of Small Business Management, 42, 3 (2004) 279-302; COMISIÓN EUROPEA, Drivers..., o.c.

${ }^{34}$ LEONIDOU, L. C., "An analysis...”, o.c. 
Las condiciones internas, como los recursos disponibles y la estrategia de marketing, son cruciales en la supervivencia exportadora. Los condicionantes internos son los que pueden ser controlados o influenciados por las empresas. Dentro de ellos hay dos categorías: los relacionados con los recursos y los relacionados con el marketing.

Los recursos que las empresas tienen que comprometer para la exportación son un factor y una limitación que hay que tener en cuenta en la actividad internaciona ${ }^{35}$. Los factores relacionados con los recursos son de tipo operativo y suelen aparecer por ineficiencia de varias funciones dentro de las empresas. Entre ellos se encuentran condicionantes como la cultura empresarial, el conocimiento, los recursos económicos, humanos, de producción y tamaño empresarial.

Los problemas relacionados con las variables del marketing aparecen fundamentalmente en las fases iniciales de la exportación. Hay muchas fuerzas que influyen en el marketing internacional de una empresa, creando barreras a la exportación que pueden ser superadas mediante las herramientas propias del marketing ${ }^{36}$.

\subsection{Dos actitudes frente a la internacionalización: proactiva y reactiva}

Como en cualquier ámbito empresarial, en la internacionalización se puede proceder de forma proactiva o reactiva. En el reto de la internacionalización la actitud de la empresa es crucial para el éxito.

En líneas generales la actitud reactiva es propia de empresas tradicionales, entendiendo como tales las empresas que primero se desarrollan en su propio país y, en un momento dado, se expanden al nivel internacional. Siguiendo el enfoque del modelo de internacionalización de la Escuela Uppsala, primero acceden a mercados cercanos y se alejan paulatinamente, marcando diferentes etapas $^{37}$. No obstante, este modelo ya ha sido superado en muchos casos, como ocurre con las empresas que nacen directamente con vocación internacional. Ambos modelos coexisten actualmente y no hay uno mejor que otro.

Por otra parte, la actitud proactiva se asocia a empresas recién creadas que salen directamente al exterior, sin tener que pasar por un periodo de madurez

${ }^{35}$ ARTEAGA, J., La actividad exportadora ..., o.c.

${ }^{36}$ LEONIDOU, L. C., "An analysis...", o.c.

37 VAHLNE, J. F., y JOHANSON, J., "The Uppsala model...", o.c.; JOHANSON, J., y WIEDERSHEIM-PAUL, F., "The internationalisation of the firm: four Swedish cases", in Journal of Management Studies, 12, 3 (1975) 305-322. 
en el mercado doméstico. También es el caso de empresas que inician su andadura internacional de forma reactiva, pero cambian de estrategia con el tiempo. La actitud proactiva se relaciona con la existencia en la empresa de un plan de internacionalización ${ }^{38}$.

Desde el prisma de la exportación se puede afirmar que estas dos actitudes están relacionadas con las causas que provocaron el inicio de la actividad internacional de la empresa: la motivación.

La motivación reactiva u oportunista es común en algunos sectores en los que la actividad internacional se inicia por ser proveedor de algún gran cliente con actividad internacional. Ante la situación de que el cliente tenga actividad internacional, el proveedor no tiene más remedio que internacionalizarse para no perder su cliente. La imitación de otros competidores es otra forma de motivación reactiva que ha sido común en sectores como en la alimentación. Por último, la actividad exportadora puede ser desencadenada por factores como un pedido puntual del extranjero o a consecuencia de un contacto importante ${ }^{39}$. Todas estas circunstancias reactivas no han sido intencionadas ni programadas con anterioridad, por lo que su sostenibilidad vendrá condicionada por otros factores y no estará tan controlada por la empresa como podría estarlo en el caso de una motivación y actitud proactivas, intencionadas.

Ineludiblemente el hecho histórico de la crisis económica (2008) tuvo una fuerte influencia en la actitud reactiva u oportunista de muchas empresas. Hay tres subgrupos de empresas en la historia reciente de la internacionalización española. Un primer grupo aprovechó bien el periodo de crecimiento y fueron previsores, diversificando y ampliando su mercado más allá de las fronteras nacionales. Un segundo grupo lo conformaban empresas con unas cuentas saneadas que pudieron afrontar la internacionalización cuando el mercado interno cayó. En la cola se encontraban empresas que sufrieron mucho por la crisis económica. Estas vieron en la internacionalización una tabla de salvación ${ }^{40}$.

La decisión proactiva o vocación exportadora se hace en base a una planificación estratégica de antemano, apoyada en una decisión científica teniendo en cuenta una serie de variables internas y externas. Aunque la internacionalización derive de una decisión y actitud activa, se debe conocer y aplicar una metodología

\footnotetext{
${ }^{38}$ VARIOS, "Towards an integrative model of small firm internationalization", in Journal of International Entrepreneurship, (2003) 339-362; CARNICER, D., Plan de internacionalización ..., o.c.

${ }^{39}$ VARIOS, "Marketing internacional...", o.c.

${ }^{40}$ REQUEIJO, J., "La competitividad de la economía..., o.c.; PERETE, J., "Internacionalizarse a lo loco", en El futuro se decide ahora. Ideas para mejorar tu empresa, Valencia, Romeditors, 2013, pp. 118-123.
} 
adecuada para emprender con éxito el camino hacia mercados internacionales. La planificación estrategica de la posible internacionalización es una pieza fundamental para el éxito de la misma ${ }^{41}$.

El momento en que una empresa empieza su actividad internacional es clave. En la historia reciente destaca un periodo concreto que influyó marcadamente en el desarrollo de la internacionalización de las empresas. Fue la crisis de $2008^{42}$.

Una primera clasificación por etapas para el caso español podría ser la siguiente: del año 2000 al 2007, que fue una etapa de bonanza y crecimiento; del 2008 al 2014, cuando algo más de 300.000 pymes desaparecieron de la esfera económica española; y del 2015 al 2019, cuando el mercado interno se empezó a reactivar ${ }^{43}$.

En terminos generales, entre el 2000 y el 2007, la salida al exterior de las empresas españolas no se debió a problemas en el mercado interno, excepto en algunos sectores concretos, sino al deseo de las pymes de expandir su mercado.

En el segundo periodo (2008-2014), hubo un cambio de tendencia que respondía principalmente a la salida al exterior de empresas que antes se conformaban con el mercado nacional. La crisis y la falta de demanda interna las animaron a salir para buscar mayores beneficios ${ }^{44}$. En general, las empresas que se internacionalizaron en este periodo lo hicieron motivadas por la crisis económica doméstica, la bajada del consumo nacional y la necesidad de vender la producción en otros mercados. Esto dio lugar a dos grupos de empresas: las que empezaron a exportar y vieron posibilidades que antes no habían contemplado; y las que solo buscaron una solución temporal al problema doméstico. La exportación constituyó un auténtico salvavidas durante la crisis ${ }^{45}$.

Entre 2008 y 2014, los programas de iniciación y apoyo a la exportación que habían empezado antes de la crisis continuaron. También aparecieron otros programas para animar a pymes con productos exportables a vender fuera de España y mitigar así el descenso del consumo nacional. Con el tiempo estos últimos programas se fueron especializando por sectores, fases de interna-

\footnotetext{
${ }^{41}$ CARNICER, D., Plan de internacionalización..., o.c.; VARIOS, "Marketing internacional...", o.c.

${ }^{42}$ REQUEIJO, J., "La competitividad de la economía...”, o.c.

${ }^{43}$ VARIOS, "El impacto de la crisis económica en las pymes", en Economistas, 149 (2016) 66-80.

${ }^{44}$ PEÑA, J., "Las exportaciones españolas de servicios: mitos y realidades", en Economistas, 148 (2016) 49-65.

${ }^{45}$ PERETE, J., "Internacionalizarse..., o.c. 
cionalización, factores geográficos, tamaño y organización de las empresas. Del compromiso de la gerencia y recursos disponibles de cada empresa ha dependido en gran parte la continuidad o abandono de la actividad internacional. La actitud reactiva o proactiva de las empresas frente a la exportación demuestra la existencia de una percepción distinta de las barreras y condicionantes a la exportación a las que la empresa tiene que hacer frente durante su actividad internacional.

La exportación ha mejorado la competitividad de las empresas españolas, internacionalizando sus actividades y reestructurando la maquinaria productiva del país. Esto ha provocado un cambio en la percepción del proceso de internacionalización. Ahora se considera una necesidad y no solo una posibilidad. La internacionalización hace que las empresas resulten más atractivas a clientes, proveedores y posibles aliados estratégicos ${ }^{46}$.

\subsection{Efectos de la internacionalización}

Existe un consenso general sobre los beneficios del comercio internacional de bienes y servicios tanto para la empresa involucrada como para el crecimiento económico de un país. Sin embargo, hay que señalar que los beneficios no siempre se quedan en el país de origen. Es el caso de la repatriación de los beneficios de empresas extranjeras ${ }^{47}$.

El volumen de exportación y el grado de internacionalización de las empresas inciden de manera importante en el nivel de desarrollo económico de un país. El ICEX cuantifica un aumento de ventas en el exterior de un $71,1 \%$ por parte de las empresas que han participado en su Plan de Iniciación a la Exportación (PIPE 2000) en tan solo dos años y medio. Este programa, impulsado por las Cámaras de Comercio y el Instituto Español de Comercio Exterior, ha tenido continuidad en el tiempo. La mayoría de las 3.050 empresas participantes eran pymes ${ }^{48}$. Entre los resultados destaca que la facturación media debida a la exportación ha pasado de 323.600 euros a 553.800 y que ha habido un aumento de la plantilla de las empresas del 13,5\% durante la duración del programa $^{49}$. No obstante, hay que tener en cuenta que el éxito de programas como el PIPE 2000 está influenciado por factores ajenos al propio programa.

\footnotetext{
46 VARIOS, Alianzas Inteligentes para la transformación competitiva de las organizaciones, Madrid, McGraw-Hill, 2020; BONET, A., "La nueva Cámara...”, o.c.

${ }^{47}$ CALVO, A., Economía internacional..., o.c.

48 ARTEAGA, J., y MEDINA, D.R., "La importancia del tamaño en la actividad exportadora: una evaluación en el caso de las PYMEs españolas no consolidadas”, en Boletín Económico ICE, Información Comercial Española, 2883 (2006) 41-54.

${ }^{49}$ ICEX, 2005. Recuperado el 22 de agosto de 2021, de http://www.comercio-exterior.es
} 
La presencia de exportadores consolidados facilita la salida al exterior de empresas que en un principio están orientadas al mercado doméstico. A parte de los beneficios que recibe la propia empresa por internacionalizarse, es importante remarcar que, al hacerlo, anima a otras a seguir su ejemplo. De este modo aumenta el número de beneficiados por la internacionalización.

Además, la actividad exportadora de una empresa o sector concreto en un país tiene un efecto positivo sobre la imagen exportadora de dicho país ${ }^{50}$.

La internacionalización también tiene un efecto positivo en la logística. Cuando una zona empieza a exportar tiene lugar un desarrollo logístico en ella. Esto facilitará la aparición de nuevos exportadores en dicha zona.

Los efectos de la internacionalización en la propia empresa dependerán de cómo se enfoque este proceso. Será de gran importancia que se haya prestado la debida atención a factores como entender el país de destino, conocer el marco legal y adaptar el producto al consumidor internacional. Es vital establecer una estrategia clara de entrada en los países o mercados.

En las primeras fases de la internacionalización y diversificación geográfica no se obtiene un beneficio palpable. En ellas es crucial que los directivos sean proactivos para enfrentarse a actividades nuevas y complejas. Las empresas sin esta aspiración de diversificar mercados tienden a protegerse. Esto los lleva en algunos casos a fracasar y volver a sus mercados domésticos. La diversificación geográfica y el papel de los directivos es una de las causas de la supervivencia exportadora. Cuando una empresa se ha consolidado como exportadora se hace más productiva e innovadora ${ }^{51}$. Además, se puede ver un aumento de las habilidades profesionales ${ }^{52}$.

Los efectos de la internacionalización en las empresas son diferentes en función de si cuentan con una trayectoria comercial anterior a su internacionalización, como suele ser el caso de las empresas tradicionales que siguen el modelo Uppsala; o bien si son empresas creadas para salir directamente al exterior. Para las primeras, que suelen ser las más comunes, destaca el incremento del volumen de ventas, la ampliación de la cuota de mercado, la extensión del ciclo de vida del producto y, sobre todo, su crecimiento y supervivencia. Para las creadas para salir directamente al exterior, los efectos de su temprana

\footnotetext{
${ }^{50}$ KRUGMAN, P. R., \& OBSTFELD, M., Economía internacional ..., o.c.

${ }^{51}$ VRONTIS, D., y CHRISTOFI, M., "R\&D internationalization and innovation: A systematic review, integrative framework and future research directions", in Journal of Business Research, 128 (2021) 812-823.

${ }^{52}$ COMISIÓN EUROPEA, Drivers of SME internationalization ..., o.c.
} 
internacionalización son la obtención de una ventaja competitiva, la rápida penetración en el mercado global tanto en nichos como en segmentos y la protección de sus conocimientos ${ }^{53}$. En este último caso es destacable el impacto de la actitud proactiva hacia la internacionalización por parte de las empresas desde el primer momento.

Por otra parte, la presencia en diferentes mercados obliga a las empresas a adaptarse a mayor cantidad y variedad de feedback. Esto constituye un incentivo para que aumenten su inversión en investigación y desarrollo ${ }^{54}$.

Estas inversiones son se justifican y compensan por el beneficio que se obtiene en los mercados internacionales.

La internacionalización de una empresa también tiene un impacto directo en aspectos intangibles como el prestigio en el mercado doméstico. Además, la hace más atractiva para profesionales especializados ${ }^{55}$.

Sin embargo, aunque en general se considera que la internacionalización tiene efectos positivos, no siempre es así, ni siempre son los mismos. Existen una serie de errores cometidos habitualmente por las empresas en el comienzo de su proceso de internacionalización como no planificar la exportación, falta de continuidad, errores de marketing y comercialización. El fracaso exportador tiene como consecuencias la pérdida del mercado objetivo y, especialmente, una mala experiencia que inhibirá la internacionalización más adelante ${ }^{56}$.

Así, se puede intuir que los efectos de la internacionalización para las pymes son casi siempre positivos y tienden a crecer más rápidamente que empresas equivalentes que operan solo en el mercado nacional ${ }^{57}$. Pero el exportador debe tener una clara voluntad de exportar, alcanzar una dimensión de empresa suficiente y, por último, definir una estrategia empresarial y de marketing clara y por escrito enfocada a la diversificación de mercados internacionales.

\footnotetext{
${ }^{53}$ VARIOS, Towards..., o.c.

${ }^{54}$ SECRETARÍA DE ESTADO DE COMERCIO, Plan de acción para la internacionalización... o.c.

${ }^{55}$ JORGE-MARTÍN, R., "El arraigo territorial en la internacionalización...”, o.c.

${ }^{56}$ PERETE, J., «Internacionalizarse...”, o.c.; CARNICER, D., Plan de internacionalización..., o.c.

${ }^{57}$ CARNICER, D., Price in international ..., o.c.
} 


\section{CONCLUSIONES}

La internacionalización es un proceso clave en la expansión de la empresa. A lo largo del tiempo ha pasado de ser un proyecto dirigido por los estados a ser una iniciativa propia de la empresa, siempre con el apoyo de dichos Estados. Es preciso diferenciarla de la exportación, que se reduce a la venta en mercados extranjeros y constituye solo una expresión mínima del proceso de internacionalización.

Hay muchas razones para la internacionalización de las empresas, pero la principal es la búsqueda de beneficios por medio de la apertura a nuevos mercados. En la internacionalización, las empresas se enfrentan a barreras y condicionantes que dificultan y marcan el proceso. La diferenciación entre externas e internas, en función del control que la empresa tiene sobre ellas, permite desarrollar la base de estrategias para la superación o adaptación a cada una de dichas barreras.

El aspecto fundamental a tener en cuenta para iniciar la actividad internacional, y especialmente para la supervivencia exportadora, es la actitud de la empresa. Es necesaria una actitud proactiva para superar las dificultades de la internacionalización.

La internacionalización tiene, por lo general efectos positivos para las empresas. Permite un aumento de los beneficios y una diversificación del riesgo, incrementando la experiencia y conocimientos de la empresa. Además, la internacionalización tiene un impacto positivo directo sobre la imagen de la empresa.

La planificación de la internacionalización, imprescindible para el éxito y supervivencia internacional de la empresa, exige un conocimiento del proceso lo más amplio y preciso posible.

\section{BIBLIOGRAFÍA}

- AABY, N., y SLATER, S.F.., "Management influences on export performance: a review of the empirical literature 1978-1988", in International marketing review, 6, 4 (1989) 7-26.

- ARTEAGA, J., La actividad exportadora y las barreras a la exportación. Una aplicación empírica a las pequeñas y medianas empresas españolas. Tesis doctoral, Universidad de Las Palmas de Gran Canaria, 2003. 
- ARTEAGA, J., y MEDINA, D.R., "La importancia del tamaño en la actividad exportadora: una evaluación en el caso de las PYMEs españolas no consolidadas", en Boletín Económico ICE, Información Comercial Española, 2883 (2006) 41-54.

- BILKEY, W. J. y G. TESAR., "The export behavior of smaller-sized Wisconsin manufacturing firms", in Journal of International Business Studies, 8, 1 (1977) 93-98.

- BONET, A., "La nueva Cámara de Comercio de España y la internacionalización empresarial", en Economistas, 148 (2016) 157-165.

- CALVO, A., Economía internacional y organismos económicos internacionales, 2. Madrid, Editorial Universitaria Ramón Areces UNED, 2016.

- CARNICER, D., Plan de internacionalización de empresas. Valencia: Centros Europeos de Empresas Innovadoras de la Comunidad Valenciana (CEEI CV), 2011.

- CARNICER, D., Price in international marketing, Madrid, ESIC Editorial, 2016.

- CARNICER, D., "Claves para la Internacionalización de la Empresa Familiar", en Cuadernos de Trabajo de la Cátedra de Empresa Familiar de la Universitat de València, Valencia 2020.

- CAVUSGIL, S. T., Organizational determinants of the firm's export behavior: an empirical analysis. Ph. D. dissertation. The University of Wisconsin, 1976.

- COMISIÓN EUROPEA, Drivers of SME internationalization, 2014.

- CRICK, D., "The decision to discontinue exporting: SMEs in two U.K. trade sectors", in Journal of Small Business Management, 40, 1 (2002) 66-77.

- EDGEWORTH, F. Y., Mathematical Psychics an Essay on the Application of Mathematics to the Moral Sciences, Forgotten Books, 1881/2012.

- GARRIDO BUJ, S., Manual de gestión de empresas, Madrid, Editorial Universitas UNED, 2011.

- HABERLER, G., The theory of international trade, London, Willian Hodge, 1936. 
- HECKSCHER, E. F., y OHLIN, B.G., Heckscher-Ohlin trade theory, The MIT Press, 1991.

- HOLLENSEN, S., y ARTEAGA, J., Estrategia de marketing internacional, 4. Madrid, Pearson Educación, 2010.

- IETTO-GILLIES, G., "Conceptual issues behind the assessment of the degree of internationalization", in Transnational Corporations, 18, 3 (2009).

- ICEX-CECO, Manual de internacionalización, 2. Madrid, AEBOE, 2017.

- JOHANSON, J., y WIEDERSHEIM-PAUL, F., "The internationalisation of the firm: four Swedish cases", in Journal of Management Studies, 12, 3 (1975) 305-322.

- JOHANSON, J., y VAHLNE, J. F., "The internationalisation process of the firm. A model of knowledge development and increasing foreign market commitments", in Journal of International Business Studies, 8,1 (1977) 23-32.

- JOHNSON, H.G., International trade and economic growth: studies in pure theory, Harvard University Press, 1958.

- JONES, R. W., y NEARY, J. P., "The positive theory of international trade", in JONES, R. W., y KENEN, P. K., Handbook of international economics, Vol. 1. Amsterdam, North-Holland, 1984.

- JORGE-MARTÍN, R., "El arraigo territorial en la internacionalización del vino español", en Anuario Jurídico y Económico Escurialense, 53 (2020) 353-378.

- KRUGMAN, P. R., y OBSTFELD, M., Economía internacional, 7. Madrid, Pearson Educación, 2006.

- LANCASTER, K., "Intra-industry trade under perfect monopolistic competition", in Journal of International Economics (1980) 151-175.

- LEONIDOU, L. C., "An analysis of the barriers hindering small business export development", in Journal of Small Business Management, 42, 3 (2004) 279-302.

- LEONTIEF, W. W., "The use of indifference curves in the analysis of foreign trade", in The quarterly Journal of Economics, 47, 3 (1933) 493-503. 
- MARSHALL, A., Principles of Economics $8^{\text {a }}$. London, MacMillan, 1890/ 1920.

- MILL, J. S., Principles of Political Economy, New York, D. Appleton and Company, 1848/1885.

- MUN, T., La riqueza de Inglaterra por el comercio exterior, México, Fondo de Cultura Económica, 1664/1978.

- PEÑA, J., "Las exportaciones españolas de servicios: mitos y realidades", en Economistas, 148 (2016) 49-65.

- PERETE, J., "Internacionalizarse a lo loco. El futuro se decide ahora. Ideas para mejorar tu empresa". Valencia. Romeditors, 2013, pp. 118-123.

- PIGAFETTA, A., La primera vuelta al mundo: Relación de la expedición de Magallanes y Elcano (1519-1522), Madrid, Alianza Editorial, 2019.

- PORTER, M. E., Ventaja competitiva, Madrid, Alay, 2002.

- REQUEIJO, J., "La competitividad de la economía española (20082018)", en Economistas, 166 (2020) 31-36.

- RICARDO, D., Principios de Economía Política y Tributación, Madrid, Ediciones Pirámide, 1817/2003.

- SAMUELSON, P. A., "The gains from international trade one again", in Economic Journal, 72.288 (1962) 820-829.

- SCHUMPETER, J. A., Theorie der wirtschaftlichen Entwicklung, Leipzig, Verlag von Duncker und Humblot, 1911.

- SECRETARÍA DE ESTADO DE COMERCIO, Plan de acción para la internacionalización de la economía española 2021-2022, Madrid, CPAGE, 2020.

- SMITH, A., La riqueza de las naciones, Madrid, Alianza Editorial, 1776/ 1997.

- STOLPER, W. F., y SAMUELSON, P.A., "Protection and real wages", in The review of economic Studies, 9, 1 (1941) 58-73.

- TAMAMES, R., "Sin alternativa a la globalización", en Revista de Economía Mundial, 18 (2008) 126-139. 
- VAHLNE, J. F., y JOHANSON, J., "The Uppsala model: Networks and microfundations", in Journal of International Business Studies, 51, 1 (2020) 4-10,

- VARIOS, "Towards an integrative model of small firm internationalization", in Journal of International Entrepreneurship, (2003) 339-362.

- VARIOS, "Explaining intermittent exporting: exit and conditional re-entry in export markets", in Journal of International Business Studies, 47, 9 (2016) 1058-1076.

- VARIOS, "El impacto de la crisis económica en las pymes", en Economistas, 149 (2016) 66-80.

- VARIOS, Marketing internacional. Manual de internacionalización, 2. Madrid, ICEX España, Exportación e Inversiones y el Centro de Estudios Económicos y Comerciales, 2017, pp. 218-372.

- VARIOS, Alianzas Inteligentes para la transformación competitiva de las organizaciones, Madrid, McGraw-Hill, 2020.

- VRONTIS, D., y CHRISTOFI, M., "R\&D internationalization and innovation: A systematic review, integrative framework and future research directions", in Journal of Business Research, 128 (2021) 812-823. 